\title{
Metastatic Merkel cell carcinoma and myasthenia gravis: contraindication for therapy with immune checkpoint inhibitors?
}

Anne Zaremba ${ }^{1 *}$, Eleftheria Chorti ${ }^{1}$, Finja Jockenhöfer ${ }^{1}$, Saskia Bolz ${ }^{2}$, Selma Sirin ${ }^{3}$, Martin Glas ${ }^{4}$, Jürgen C. Becker ${ }^{1,5}$, Selma Ugurel', Alexander Roesch', Dirk Schadendorf ${ }^{1}$, Elisabeth Livingstone ${ }^{1}$, Tim Hagenacker ${ }^{2+}$ and Lisa Zimmer ${ }^{1 *+}$

\begin{abstract}
Background: PD-1/PD-L1 inhibitors are promising approaches for advanced Merkel cell carcinoma (MCC). Nevertheless, these inhibitors bear a high risk for induction of immune-related adverse events (irAEs), particularly flares of preexisting autoimmune diseases. Neurological irAEs of PD-1/PD-L1 inhibitors are possibly underestimated and potentially fatal toxicities. Additionally, exacerbations of preexisting myasthenia gravis (MG) with a high MGspecific-related mortality have been reported.

Case presentation: A 61-year-old woman with a history of MG since 2005 was treated with azathioprine and pyridostigmine after thymectomy. In March 2016, she was diagnosed with MCC. Six months later the tumor had progressed to stage IV and metastases were detected in lymph nodes and the pancreas. The immunosuppressive therapy was therefore changed to mycophenolatmofetil (MMF) and an immune checkpoint blockade with the PD-1 inhibitor pembrolizumab was initiated in November 2016. Due to MMF-induced liver toxicity, MMF was switched to cyclosporine A (CSA) with normalized liver transaminases six weeks later. After six cycles of pembrolizumab the patient achieved a partial response. Follow up analysis sixty-five weeks later revealed a long-lasting tumor response with a partial remission of pancreatic and inguinal metastases and no flare of MG.

Conclusions: Patients with a preexisting MG can be considered for treatment with immune checkpoint inhibitors if they have a life-threatening cancer and if other effective, long-lasting treatment options are not available. The risks and benefits of therapy should be weighed in a multidisciplinary setting and should be discussed thoroughly with the patient. Exacerbation of underlying MG can be potentially life-threatening and requires close monitoring in collaboration with neuromuscular specialists.
\end{abstract}

Keywords: Merkel cell carcinoma, Myasthenia gravis, Immune checkpoint inhibitor, Adverse events, Immunotherapy

\footnotetext{
* Correspondence: anne.zaremba@uk-essen.de; lisa.zimmer@uk-essen.de Tim Hagenacker and Lisa Zimmer are shared authorship

${ }^{1}$ Department of Dermatology, University Hospital Essen, Essen, Germany

Full list of author information is available at the end of the article
}

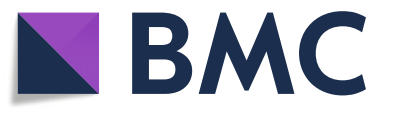

(c) The Author(s). 2019 Open Access This article is distributed under the terms of the Creative Commons Attribution 4.0 International License (http://creativecommons.org/licenses/by/4.0/), which permits unrestricted use, distribution, and reproduction in any medium, provided you give appropriate credit to the original author(s) and the source, provide a link to the Creative Commons license, and indicate if changes were made. The Creative Commons Public Domain Dedication waiver (http://creativecommons.org/publicdomain/zero/1.0/) applies to the data made available in this article, unless otherwise stated. 


\section{Background}

Blocking antibodies for programmed cell death protein 1 (PD-1) are commonly used for the treatment of metastatic melanoma and other tumours[1-3]. Although advanced Merkel cell carcinoma (MCC) responds to chemotherapy, responses are seldom durable, showing a median progression-free survival of only 94 days [4]. As MCC cells often express programmed cell death protein ligand 1 (PD-L1) and Merkel cell polyomavirus (MCPyV)specific $\mathrm{T}$ cells express corresponding PD-1, blockage of the PD-1 immune inhibitor pathway is of interest and PD1/PD-L1 inhibitors have been shown to be a promising approach for the treatment of advanced MCC [5, 6]. Recently, three phase II open-label clinical trials of the PD-1/ PD-L1 inhibitors pembrolizumab, nivolumab and avelumab in patients with metastatic MCC have demonstrated high and durable response rates of 57, 73 and $62.5 \%$, respectively [5-7]. Nevertheless, PD-1/PD-L1 inhibitors also bear the risk for inducing immune-related adverse events (irAEs). The most frequent irAEs are skin toxicities, colitis, hepatitis and endocrinopathies [1]. Rare irAE include pneumonitis, nephritis, neurological and cardiological side-effects. Neurologic irAEs of the central and peripheral nervous system (PNS) have been reported in up to $12 \%$ of patients treated with immune checkpoint inhibitors [8-10]. Common neurologic irAEs of the PNS include mild to moderate peripheral neuropathies, but cases of life-threatening and fatal cases of Guillain-Barré syndrome, necrotizing myositis and myasthenic syndromes have been reported $[7,8]$. In the literature, 23 cases of MG after immunotherapy with checkpoint inhibitors have been described, the majority being de novo cases (72.7\%), but also some cases of exacerbations of a preexisting MG (18.2\%) or subclinical MG (9.1\%) [1]. MG-related mortality was estimated at $30.4 \%$ [1]. Only limited experience exists regarding therapy with immune-checkpoint inhibitors in patients with preexisting autoimmune disorders, as they are often excluded from clinical trials [11].

In this case report, we describe our recent experience with administration of pembrolizumab in a patient with metastatic MCC and well-controlled MG on immunosuppressive therapy.

\section{Case presentation}

A 61-year-old woman was diagnosed with anti-acetylcholine receptor antibody (ACh-R) positive MG in 2005. Initially, only ocular signs were present, but systemic symptoms arose over time showing a relapsing course. During her last myasthenic crisis in 2009 a thymectomy was performed and an immunosuppressive therapy with azathioprine in combination with pyridostigmine was initiated. Neurological symptoms were fully controlled without residual symptoms. Doses of azathioprine and pyridostigmine remained stable during the regular three-monthly neurologic screening visits.
In March 2016 a MCPyV-positive MCC measuring $>5 \mathrm{~cm}$ in diameter with a tumor thickness of $22 \mathrm{~mm}$ was detected on her right gluteal side. After wide local excision of the primary tumor with a $3 \mathrm{~cm}$ safety margin and a negative sentinel lymph node biopsy of the right groin, she received an adjuvant radiotherapy of the primary tumor site. The patient underwent a rigorous follow-up scheme with clinical examinations and ultrasound of the regional lymph nodes every six weeks and yearly chest X-ray and abdominal ultrasound were planned. In September 2016, six months after the initial diagnosis of MCC, ultrasound of the right inguinal groin showed enlarged lymph nodes. A subsequent positron emission tomography (PET)-computed tomography (CT) confirmed right inguinal lymph node metastases. Additionally, metastases of the pancreatic tail and its surrounding lymph nodes were detected. To exclude a secondary malignancy, a biopsy from the pancreas was performed confirming MCC metastasis. Due to the extensive metastatic spread of the MCC, immune-checkpoint therapy with a PD-1 inhibitor was recommended by our interdisciplinary tumor board. The risks (i.e. exacerbation of preexisting MG with potential lethal outcome) and benefits (i.e. life-threatening metastatic MCC with a response rate of around 60\% to PD-1/PD-L1 inhibitors) of PD-1/PD-L1 inhibitor therapy was discussed thoroughly with our neuromuscular specialists and the patient. As treatment with azathioprine has been identified as a risk factor for the development of non-melanoma skin cancer in transplant recipients and myasthenia patients [12-15] therapy for MG was switched from azathioprine to mycophenolatmofetil (MMF) (500 mg 1-0-1). After extensive education of the patient and the patients' family, immunotherapy using PD-1 inhibitor pembrolizumab at a dose of 2 $\mathrm{mg} / \mathrm{kg}$ every three weeks was started in November 2016. At the time of therapy initiation avelumab was neither approved for MCC nor available to our skin cancer unit [7]. Therefore, due to published overall response data at that time [6], pembrolizumab was chosen.

Prior to initiation of immunotherapy, screening laboratories for hepatitis A, B and C, were performed and negative. The patient had no medical history for hepatotoxicity in the past, therefore marginally elevated transaminases were tolerated at start of PD-1 inhibitor therapy. Due to parallel induction of MMF and pembrolizumab, blood levels including liver enzymes were measured more frequently than normal. After one cycle of pembrolizumab, the patient's liver enzymes started to rise slightly (grade 1 common toxicity criteria of adverse events (CTCAE; version 4.0)). The case was discussed in a multidisciplinary team meeting with gastroenterologists and neurologists, concluding that an immunerelated hepatitis would rather be unlikely while treated with MMF suspecting MMF-induced toxic liver damage as the most likely diagnosis. At this time, a liver biopsy was not performed due to asymptomatic grade 1 hepatitis. Since levels of aspartate aminotransferase increased on day 15 and were 
still elevated on day 21, the second pembrolizumab infusion was paused as a precaution. In addition, MMF was switched to CsA at a dose of $2 \mathrm{mg} / \mathrm{kg}$. Within two weeks, after a slight increase of the liver enzymes, transaminases decreased to initial values (Fig. 1) and pembrolizumab was continued.

Eight weeks after the start of immunotherapy with pembrolizumab, but after only two infusions, the first regular staging with CT scans of the chest, abdomen and brain revealed a mixed response with partial response (PR) of the pancreatic tail tumor and progression of the lymph node metastases of the right groin. As the tumor of the right groin caused severe pain, surgery or localized radiation therapy was recommended. However, after the third course of pembrolizumab the patient reported significant decrease of pain, clinical examination could confirm dramatic regression of the inguinal lymph nodes. Therefore, immunotherapy continued without any further local treatment to the right groin. The patient was in constant surveillance by neurology specialists using the quantitative myasthenia gravis (QMG) score incl. measurement of vital capacity every 3 months and did not experience an exacerbation of MG at any time. She remained on treatment with CsA 125 $\mathrm{mg} /$ day and pyridostigmine $60 \mathrm{mg}$ three times daily. The second staging after 23 weeks (six doses) showed further remission of the pancreatic metastasis and distinct remission of the lymph node metastases. So far, immunotherapy with pembrolizumab is still ongoing (27 doses) and CT-imaging of the abdomen revealed a persistent tumor regression of the right inguinal lymph node metastases and a no longer detectable metastasis at the pancreatic tail (Fig. 2) for 65 weeks without any flare of the MG.

\section{Discussion and conclusion}

MCC is a highly aggressive skin cancer which is linked to MCPyV or ultraviolet (UV) radiation [4, 5]. Several risk factors have been identified, primarily immunosuppression, higher age, cumulative UV radiation and other cutaneous tumors, including melanoma [5]. Lately, immunotherapy with PD-1/PD-L1 inhibitors demonstrated a promising approach for metastatic MCC with high and durable response rates ranging between 30 to $60 \%$ in the second or first line setting, respectively [5]. Notably, chemotherapy, while inducing high response rates, is not improving overall survival since responses are of very short duration [4].

As PD-1 along with CTLA-4 signaling pathways have been shown to play roles in the maintenance of selftolerance and modulation of immune responses, irAEs are expected to generate due to an imbalance in the response of $\mathrm{T}$ cells to antigens presented by normal cells [16]. Therefore, immune checkpoint inhibitors such as PD-1/ PD-L1 inhibitors are suspected to induce irAEs due to impaired self-tolerance from loss of T-cell inhibition [8-10].

In general, it can be distinguished between de novo induced neurological AEs and flares of preexisting

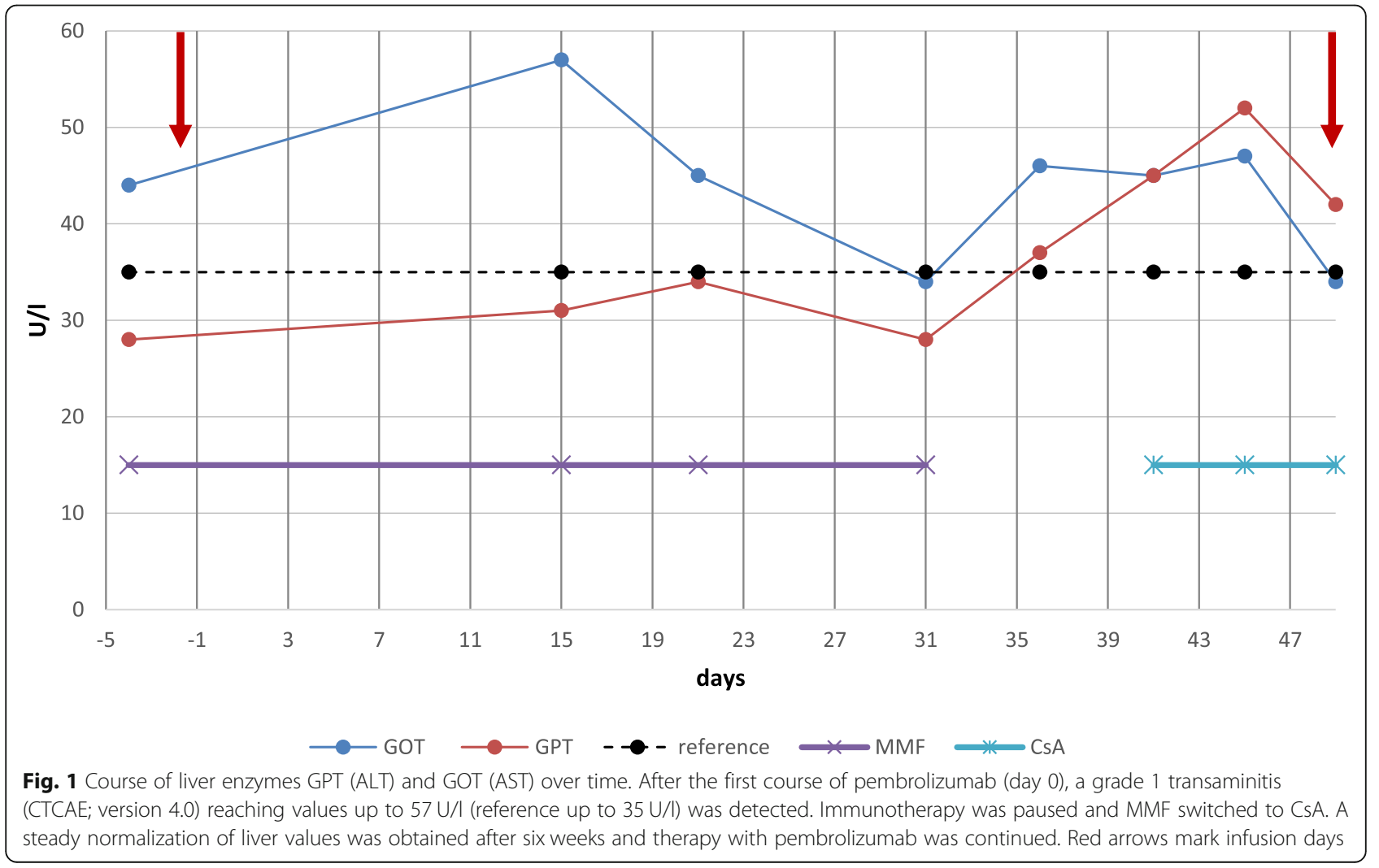



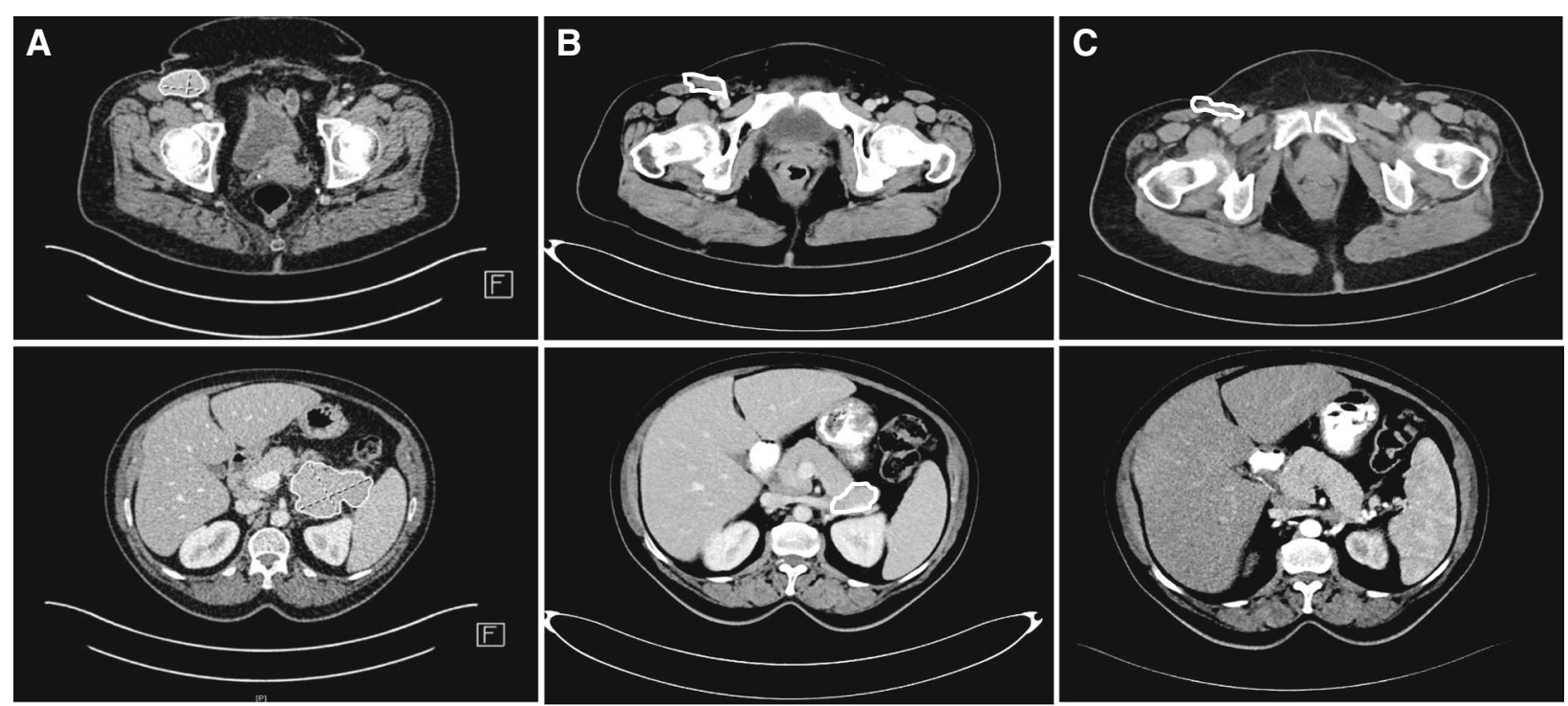

Fig. 2 The initial staging from 09/2016 (a) shows conglomerated lymph nodes at the inguinal right region and a tumor at the pancreatic tail with an increased glucose metabolism suspicious for tumorous tissue. After six courses of pembrolizumab (b) a remission of pancreatic filia and a distinct remission of lymph nodes metastases were detectable. In 02/2018 (c) the pre-described tumor at the pancreatic tail is no longer detectable and a constant consolidation at the inguinal right region can be obtained. Furthermore, no new suspicious lesions are present, showing an ongoing partial response to pembrolizumab

neurological disorders by checkpoint inhibition [2,3]. As patients with preexisting autoimmune disorders are generally excluded from clinical trials with immunotherapeutic agents, safety and efficacy data of immunotherapy in these patients is very limited and mostly of retrospective nature. In two retrospective studies including seven patients with advanced melanoma and preexisting neurological disorders (including one patient with MG, three with Guillain-Barré-syndrome, one with multiple sclerosis, one with chronic inflammatory demyelinating polyneuropathy, one with Bell's palsy) no flare occurred $[2,3]$. Currently no data exist regarding the frequency of exacerbations of preexisting MG in patients who have been treated with checkpoint inhibitors. All previously published case reports reported exacerbations of preexisting MG in patients treated with PD-1 inhibitors $[1,17,18]$. For instance, Makarious et al. reviewed existing literature on immune checkpoint inhibitorassociated MG gathering a total of 23 cases after PD-1 inhibitor, CTLA-4 inhibitor or combination therapy [1]. These patients were further divided into $72.7 \%$ (17/23) with de novo MG, $18.2 \%$ (4/23) with exacerbations of preexisting MG, and 9.1\% (2/23) with exacerbations of subclinical $M^{1}$. The mean time to onset of MG-symptoms for both de novo presentations and exacerbations was 6.95 (range 2-12) weeks after induction of pembrolizumab, 5.14 (range 2-9) weeks after nivolumab, and 4.75 weeks (range 3-6) after ipilimumab, underlining the early phase of immunotherapy as the most critical [1]. Four out of 13 patients suffering from PD-1inhibitor-induced de novo or subclinical MG died (30.8\%), while one MG-induced death was reported in the four patients who showed exacerbations of their preexisting MG after treatment with PD-1 inhibitors (25\%) [1]. Compared to this, $2 / 2$ patients treated with the combination of PD-1/PD-L1 inhibitors and CTLA-4 inhibitors died, one due to de novo immune checkpoint inhibitor induced MG, one with unknown status. These cases show that MG-specificrelated mortality was comparable between de novo or subclinical MG (30.8\%) and exacerbations of preexisting MG (25\%) after PD-1 inhibitor treatment [1]. Nevertheless, patient numbers suffering from MG, either de novo or preexisting, are small and further investigations needed to clarify risks and benefits of this patient subgroup.

Taken together, de novo or exacerbations of preexisting MG can be potentially life-threatening and should be monitored in close collaboration with neuromuscular specialists using the QMG score, incl. measurement of vital capacity. In a stable situation, we recommend a QMG scoring every three months, if symptoms get worse, clinical examination should be performed every four weeks. In case of rapid worsening of symptoms, a hospitalization should be considered. Electrophysiological measurements or antibody titers do not necessarily correlate with the clinical severity of MG, therefore repetitive measurement of titers is not helpful.

To our knowledge, currently no data exist regarding reliable factors predicting the risk of checkpoint inhibitortreated patients with underlying autoimmune diseases and there are minimal data regarding factors predisposing to a flare of the underlying autoimmune disorder. Menzies et al. reported that flares occurred more often in patients with active symptoms, in those requiring immunosuppression when 
treated with PD-1 inhibitors, and in patients with preexisting rheumatologic disorders [2]. However, patient numbers are small and results should be interpreted with caution. Furthermore, the impact of preexisting immunosuppression on the outcome of immunotherapy remains to be investigated, as so far no prospective studies testing immunosuppressive strategies have been conducted to answer this question. Nonetheless, retrospective studies have shown that patients with immunosuppressants (e.g. steroids) at start of PD-1 treatment had a lower response rate, $[2,19]$ progression-free survival, and overall survival [19] compared to those not on immunosuppressants.

Our case demonstrates that preexisting autoimmune disorders should not be considered a general contraindication for immunotherapy with PD-1 inhibitors in patients with lifethreatening metastatic cancer. PD-1 inhibitor treatment was the favorable choice for our patient, particularly in view of the patients' progressive, metastatic MCC and the known excellent response rates to PD-1/PD-L1 inhibitors [6, 7]. Nevertheless, selection of therapy and patients should consider the kind and activity of underlying autoimmune disease, as well as tumor entity and its response to PD-1/PD-L1 inhibitors compared to standard of care treatments. It should be kept in mind that the risks/benefit ratio may be less favorable for tumors with relatively low response rates to immunotherapy [20,21]. The risks and potential benefits of checkpoint inhibitor treatment as well as other therapeutic concepts should be discussed thoroughly in a multidisciplinary setting and require careful and extensive education of the patient and the patient's family. Exacerbation of underlying MG can be potentially life-threatening and should be monitored in close collaboration with neuromuscular specialists.

\begin{abstract}
Abbreviations
ACh-R: anti-acetylcholine receptor; AEs: adverse events; CsA: Cyclosporine A; CT: computer tomography; CTCAE: common toxicity criteria of adverse events; CTLA-4: cytotoxic T lymphocyte associated protein 4; ir: immunerelated; MCC: Merkel cell carcinoma; MCPyV: Merkel cell polyomavirus; MG: myasthenia gravis; MMF: mycophenolatmofetil; PD-1: programmed cell death protein 1; PD-L1: programmed cell death protein ligand 1; PET: positron emission tomography; PNS: peripheral nervous system; PR: partial response; QMG: quantitative myasthenia gravis; UV: ultraviolet
\end{abstract}

\section{Acknowledgements}

None.

\section{Authors' contributions}

AZ/LZ analyzed and interpreted the patient data and was a major contributor in writing the manuscript. All authors read and approved the final manuscript.

\section{Funding}

Not applicable.

\section{Availability of data and materials}

The datasets used and/or analysed during the current study are available from the corresponding author on reasonable request.

Ethics approval and consent to participate Not applicable.

\section{Competing interests}

A. Zaremba received travel support from Novartis and Bristol-Myers Squibb. E. Chorti received travel support from Bristol-Myers Squibb, MSD SHARP \& DOHME and Novartis.

F. Jockenhöfer declares that she has no conflicts of interest.

S. Bolz declares that she has no conflicts of interest.

S. Sirin declares that she has no conflicts of interest.

M. Glas reports a Consulting / advisory role to Roche, Novartis, Daiichi

Sankyo, Novocure and travel support from Medac and Novocure.

Furthermore he received Honoraria from Novartis, Merck, Novocure, Medac, and Kyowa Kirin.

J.C.Becker has received speaker honoraria from Amgen, MerckSerono, Pfizer, and Sanofi; he has received advisory board honoraria from Amgen, CureVac, eTheRNA, Laser, Analytica, Lytix, MerckSerono, Novartis, Rigontec, Takeda and Sanofi; and he has received research funding from Alcedis, Amgen,

Boehringer Ingelheim, BMS and MerckSerono. J.C.Becker's activities with

Bristol-Myers Squibb, MerckSerono and Pfizer are related to the submitted report (therapy of advanced MCC).

S. Ugurel declares advisory board and speakers honoraria from Bristol-MyersSquibb, Merck Sharp\& Dohme and Roche, as well as grant and travel support from Bristol-Myers-Squibb, Merck Sharp \& Dohme, Roche und medac.

A. Roesch reports non-financial support from Amgen, non-financial support from Roche, non-financial support and personal fees from Merck/MSD, nonfinancial support and grants from Novartis, non-financial support and grants from BMS, non-financial support from TEVA and grants from Adtec.

D. Schadendorf reports personal fees, adboards and patient's fees from Pierre Fabre, personal fees from Amgen, personal fees from Boehringer Ingelheim, personal fees from Leo Pharma, personal fees and patients' fees from Roche, personal fees and patients' fees from MSD, personal fees from Incyte, personal fees and patients' fees from Regeneron, personal fees from 4SC, personal fees from AstraZeneca, grants, personal fees and patients' fees from $B M S$, personal fees and patients' fees from Merck-EMD, personal fees from Pfizer, personal fees and patients' fees from Philiogen, and personal fees from Array.

E. Livingstone has had intermittent advisory board relationships with Roche, BMS, Novartis and Actelion and has received travel grants and honoraria from Roche, BMS, MSD, Amgen, Novartis, Boehringer-Ingelheim, medac. T. Hagenacker has received honoraria and travel reimbursement from Alexion, CSL Behring, Novartis, Biogen, Pfizer.

L. Zimmer has served as consultant or/and has received honoraria from Roche, Bristol-Myers Squibb (BMS), Merck Sharp\& Dohme (MSD), Novartis, Merck, Sanofi, Pierre Fabre and travel support from MSD, BMS, Amgen, Pierre Fabre and Novartis.

\section{Author details}

'Department of Dermatology, University Hospital Essen, Essen, Germany. ${ }^{2}$ Department of Neurology, University Hospital Essen, Essen, Germany. ${ }^{3}$ Department of Diagnostic and Interventional Radiology and Neuroradiology, University Hospital Essen, Essen, Germany. ${ }^{4}$ Division of Clinical Neurooncology, Department of Neurology, University Hospital Essen, Essen, Germany. ${ }^{5}$ Translational Skin Cancer Research, German Cancer Consortium (DKTK), Partner Site Essen/Düsseldorf, Essen, Germany.

Received: 29 January 2019 Accepted: 17 May 2019

Published online: 29 May 2019

\section{References}

1. Makarious D, Horwood K, Coward JIG, et al. Myasthenia gravis: an emerging toxicity of immune checkpoint inhibitors. Eur J Cancer. 2017; 82:128-36.

2. Menzies AM, Johnson DB, Ramanujam S, et al. Anti PD therapy in patients with advanced melanoma and preexisting autoimmune disorders or major toxicity with ipilimumab. Ann Oncol. 2017;28(2):368-76.

3. Gutzmer R, Koop A, Meier F, et al. Programmed cell death protein-1 (PD-1) inhibitor therapy in patients with advanced melanoma and preexisting autoimmunity or ipilimumab-triggered autoimmunity. Eur J Cancer. 2017;75:24-32.

4. Iyer JG, Blom A, Doumani R, et al. Response rates and durability of chemotherapy among 62 patients with metastatic Merkel cell carcinoma. Cancer Med. 2016;5(9):2294-301.

5. Becker JC, Stang A, Nghiem P, et al. Merkel cell carcinoma. Nat Rev Dis Primers. 2017;3:17077. 
6. Nghiem PT, Bhatia S, Lipson EJ, et al. PD-1 blockade with Pembrolizumab in advanced Merkel-cell carcinoma. N Engl J Med. 2016;374(26):2542-52.

7. Kaufman HL, Russell J, Hamid O, et al. Avelumab in patients with chemotherapy-refractory metastatic Merkel cell carcinoma: a multicentre, single-group, open-label, phase 2 trial. Lancet Oncol. 2016;17(10):1374-85.

8. Zimmer L, Goldinger SM, Hofmann L, et al. Neurological, respiratory, musculoskeletal, cardiac and ocular side-effects of anti-PD-1 therapy. Eur J Cancer. 2016;60:210-25.

9. Touat M, Talmasov D, Psimaras D, et al. Neurological toxicities associated with immune-checkpoint inhibitors. Curr Opin Neurol. 2017;30(6):659-68.

10. Cuzzubbo S, Javeri F, Tissier M, et al. Neurological adverse events associated with immune checkpoint inhibitors: review of the literature. Eur J Cancer. 2017;73:1-8.

11. Johnson DB, Sullivan RJ, Clark Jl, et al. Ipilimumab therapy in patients with advanced melanoma and preexisting autoimmune disorders. JAMA Oncol. 2016:2(2):234-40.

12. Pedersen $E G$, Pottegård $A$, Gaist $D$, et al. Risk of non-melanoma skin cancer in myasthenia patients treated with azathioprine. Eur J Neurol. 2014;21 (3):454-8

13. Vos M, Plasmeijer El, Rácz E, et al. Azathioprine to mycophenolate mofetil transition and risk of squamous cell carcinoma after lung transplantation. J Heart Lung Transplant. 2018;37(7):853-9.

14. Pedersen EG, Pottegård A, Gaist D, et al. Use of azathioprine for nonthymoma myasthenia and risk of cancer: a nationwide case-control study in Denmark. Eur J Neurol. 2013;20(6):942-8.

15. Perez HC, Benavides $\mathrm{X}$, Lozano $\mathrm{E}$, et al. Basic aspects of the pathogenesis and prevention of non-melanoma skin cancer in solid organ transplant recipients: a review. Int J Dermatol. 2017:56(4):370-8.

16. Pardoll DM. The blockade of immune checkpoints in cancer immunotherapy. Nat Rev Cancer. 2012;12(4):252-64.

17. Earl DE, Loochtan Al, Bedlack RS. Refractory myasthenia gravis exacerbation triggered By pembrolizumab. Muscle Nerve. 2018;57(4):E120-1.

18. Cooper DS, Meriggioli MN, Malik R, et al. Severe Exacerbation of Myasthenia Gravis Associated with Checkpoint Inhibitor Immunotherapy. J Neuromuscul Dis. 2017;4(2):169-73.

19. Arbour KC, Mezquita L, Hellmann MD, et al. Impact of baseline steroids on efficacy of programmed cell Death-1 and programmed death-ligand 1 blockade in patients with non-small-cell lung Cancer. J Clin Oncol. 2018, 36(28):2872-8.

20. Forster MD, Devlin MJ. Immune checkpoint inhibition in head and neck Cancer. Front Oncol. 2018:8:310

21. El-Khoueiry AB, Sangro B, Melero I, et al. Nivolumab in patients with advanced hepatocellular carcinoma (CheckMate 040): an open-label, noncomparative, phase 1/2 dose escalation and expansion trial. Lancet. 2017; 389(10088):2492-502.

\section{Publisher's Note}

Springer Nature remains neutral with regard to jurisdictional claims in published maps and institutional affiliations.

Ready to submit your research? Choose BMC and benefit from:

- fast, convenient online submission

- thorough peer review by experienced researchers in your field

- rapid publication on acceptance

- support for research data, including large and complex data types

- gold Open Access which fosters wider collaboration and increased citations

- maximum visibility for your research: over $100 \mathrm{M}$ website views per year

At $\mathrm{BMC}$, research is always in progress.

Learn more biomedcentral.com/submissions 